\title{
MARKET BASED TOOLS FOR MANAGING THE LIFE INSURANCE COMPANY
}

\author{
BY
}

\author{
Massimo De Felice and Franco Moriconi
}

\begin{abstract}
In this paper we present an approach to market based valuation of life insurance policies, in the spirit of the NUMAT proposed by Hans Bühlmann (2002) in an editorial in the ASTIN Bulletin. We have experienced the valuation method for more than one decade, both as a pricing procedure applied to policy portfolios of leading insurance companies, and by including the valuation principles into several actuarial teaching activities.

Our interest is mainly focused here on participating policies that in Italy are characterized by contractually binding profit sharing rules. The problem of the fair valuation of the liabilities generated to the insurer by these contracts can be conveniently addressed using the methods of contingent claims pricing. These allow to price correctly the options embedded into the policies and to implement consistent plans of asset-liability management. The approach also provides a market based measurement of the value of business in force for outstanding policy portfolios and consistent assessments of the financial risk based capitals.
\end{abstract}

\section{A NUMAT SYSTEM FROM ITALY}

In line with the suggestions expressed by Hans Bühlmann in the proposal of the NUMAT approach (Bühlmann, 2002) and also in the discussion of the article by Aase and Persson (Bühlmann, 2003a), we describe here our experience in applying and teaching the financial approach in valuation of life insurance policies in Italy.

Our approach has been focused on two main issues:

- providing a mark-to-market (fair) valuation of the outstanding liabilities of an insurance company, jointly with the appropriate measures of sensitivity to financial risk factors, e.g. interest rate risk, that are essential for implementing a consistent plan of asset-liability management;

- derive a more reliable measure of the value embedded in business in force (VBIF), including a mark-to-market valuation of the financial options embedded into the policies.

It is worth mentioning that when applied to a single policy at the issue date the approach also provides a fair methodology for profit testing. Another 
important by-product is the derivation of the financial components of the risk based capital of the outstanding portfolio. This is typically done by properly stressing the risk factors of the valuation model and then repeating the pricing procedure under the "worst case scenario".

Virtually all traditional life insurance policies in Italy provide benefits which are explicitly linked to the return of a reference fund, in which the technical reserves must be invested. The investment fund, usually referred to as "segregated fund" ("gestione separata" in Italian) is managed by the insurer under specified accounting rules. This kind of profit sharing mechanism is not discretionary for the insurer, but rather it is one of the terms of the contract. Using the language of the option pricing theory, the financial component of a policy can thus be considered as a derivative contract having the reference fund as the underlying asset. Therefore it is natural to perform the financial valuation of both the assets (the reference fund) and the liabilities (the promised benefits) using an appropriate stochastic pricing model based on the no-arbitrage principle.

In 1993-94 we applied for the first time this methodology to the valuation of a portfolio of participating policies issued by INA (Istituto Nazionale delle Assicurazioni), the so-called " $3 / \mathrm{N}$ " policies. These policies were annual premium endowments having premiums and benefits linked both to the reference fund return and to the consumer price index. The pricing of $3 / \mathrm{N}$ policies was performed using an extended Cox-Ingersoll-Ross model, including both real and nominal interest rates. As it usually happens, the indexation rule was rather complex and Monte Carlo procedures were used to implement the no-arbitrage valuation. One of the main goals was to verify if the amount of (inflation) indexed bonds held by the insurer into the reference fund was properly calibrated in order to hedge the inflation risk generated by the $3 / \mathrm{N}$ liabilities. We found that the index-linked bonds were overweighted in the investment portfolio ${ }^{1}$.

The teaching activity on these issues started in 1991 at the Scuola Normale Superiore di Pisa and at the Istituto Italiano degli Attuari ${ }^{2}$. The topics included both the theoretical foundations of the approach and the analysis of case studies, supported by explicit software procedures. The courses have been organized for the needs of the actuarial professionals, but a few places were offered on a no cost basis to academic institutions.

In July 2002, we presented the approach at the Summer School of the Groupe Consultatif Actuariel Europeen, in Milan. The lecture notes of the course (De Felice, Moriconi, 2002b) are available on the website www.GCActuaries.org/ events.html.

The valuation methodology has been coded in a software application, enforced by a corresponding database, forming the implementation of what today would be called a NUMAT-based system.

1 The results of this study were presented in the talk "Valuation of Index-Linked Life Insurance Policies with Minimum Guarantees by Stochastic Models for Real and Nominal Interest Rates" at the Conference on Recent Advances in Mathematical Finance, Cortona, May 29-June 3, 1994.

2 Courses have been held on Interest rate risk control and CIR model (1991-92); Asset-liability management in life insurance (1995-96); Management of pension funds (1997); Embedded value calculation in life insurance (2001-02). 
Early versions of this system were installed at SAI in 1997, in order to design pension plans with minimum guarantees; successively the system was enhanced and extended to all the outstanding policy portfolios of all the companies of the SAI Group, and finally of the Fondiaria-SAI Group. The system is applied both to the asset-liability management and to the control of the evolution of the embedded value and the cost of the minimum guarantees options. In 1999 the system has also been adopted by all the companies of Gruppo Reale Mutua Assicurazioni and by Cisalpina Previdenza and Roma Vita (at present merged in Fineco Vita).

Alleanza Assicurazioni is also using the system since 2000. The valuation procedures are running with monthly frequency and are used for providing management indications to the investment department and for monitoring the VBIF. The deeper understanding of the price determinants of the embedded options has stimulated the construction of new life products having a reduced cost of the minimum guarantees. These new policies have been recently issued on the market.

RAS Group adopted the methodology in 2001 in order to measure the risk based capital of the outstanding portfolios. In this case we extended the system to non-life portfolios. The embedded value measurement and the pricing of the embedded options were obtained as by-product of the risk capital valuation. Indications provided by the valuation procedures stimulated a re-styling process of the policies.

From the point of view of NUMAT approach it is important to stress that the system is employed in educational activities within the above companies.

In the rest of the paper we provide an illustration of our approach to the market based valuation of life insurance policies. When financial uncertainty (i.e. concerning capital markets) and actuarial uncertainty (e.g. concerning the life of the individuals) can be separated, the financial component of the policies can be analyzed using the tools of contingent claims pricing in complete markets. In this case the current prices of financial products contain sufficient information to derive a unique market price reflecting both the prevailing expectations and the risk premia required by the economic agents. We provide an example of how this valuation process can be realized in practice using an arbitrage pricing model including both interest rate and stock price risk. Since purely actuarial products typically are not traded in organized markets, the problem of determining a unique assessment of actuarial risk premia and hence deriving a (unique) fair valuation of the actuarial component of the policy remains an open issue ${ }^{3}$.

The idea of separating the financial from the actuarial uncertainty into an insurance contract and then of pricing the first component as a purely financial contingent claim can be traced back to Brennan-Schwartz (1976) and BoyleSchwartz (1977), who pioneered the application of the option pricing theory

\footnotetext{
3 The problem is of major importance in property/casualty insurance, where actuarial risk is usually much more relevant than financial risk. In the USA the Casualty Actuarial Society (CAS) created a "Task Force on Fair Value Liabilities" to address fair value insurance issues raised by several accounting proposals. In the "White Paper" published in 2000 by the Task Force (CAS Task Force, 2000) a number of alternative methods are considered for estimating adjustments for risk in fair valuation of actuarial liabilities. Which of these methods should be preferred is still a matter of debate.
} 
to life insurance policies. Boyle (1978) nicely applied these ideas also to the asset-liability management in insurance. These topics gained new relevance in the early 1990's, both for the strong financial innovation of the insurance products and for the decrease of interest rates experienced in the financial markets. Papers on unit-linked contracts include Delbaen (1990), Bacinello-Ortu (1993), Aase-Persson (1994). Applications of semi-deterministic and stochastic immunization theory to the asset-liability management can be found in CastellaniDe Felice-Moriconi (1992). A stream of papers followed concerning the contingent claims approach to the analysis of participating policies and to the valuation of the interest rate options embedded in these contracts. Examples are Persson-Aase (1997), Miltersen-Persson (1999), Grosen-Jørgensen (2000), Bacinello (2001), De Felice-Moriconi (2002a), Miltersen-Persson (2003).

This still growing research activity is also motivated by the position recently adopted by the international regulatory and accounting institutions, which are paying an increasing attention to the marked based assessment of the costs and the risks which are implicit in the insurance business. For example, the need to value the embedded options explicitly is widely stressed in the Research Report of the Insurer Solvency Assessment Working Party (2004) appointed by the International Actuarial Association. In this report the importance to have a consistent valuation approach for the options and their underlying assets is also recognised. Moreover, the newly developing International Accounting Standards (IAS) are clearly moving toward a fair (i.e. market consistent) valuation of insurance assets and liabilities. In particular, in the Phase II, which should be effective in 2007 or 2008, the IAS will require options and guarantees contained in insurance contracts to be explicitly valued and reserved for.

Fair valuation principles have pervasive effects on the organization of an insurance company, involving not only technical problems of pricing but also more general issues concerning accounting, capital budgeting and solvency measurement. Since we are reporting here on our experience in the practical application of these principles, we are mainly interested in questions concerning the global framework of the insurance business, as general valuation principles, reserving, embedded value and risk capital determination. More technical - though relevant - issues, as the different performances of alternative pricing models will not be of our major concern in this paper.

When considering a real-world policy portfolio a number of diffent products and contractual details has to be analyzed. To simplify the exposition we mainly refer to the simple case of pure endowment profit sharing policies. However the pricing model we specify is suited for providing a unified valuation of both the outstanding liabilities of a general policy portfolio and the assets of the fund where the earned premiums are invested.

\section{THE VALUATION FRAMEWORK}

\subsection{A simplified contract}

To understand the valuation framework it suffices to illustrate our procedure with the simple case of a single premium pure endowment insurance contract, written 
at time 0 for a life with age $x$, with term $T$ years and initial sum insured $C_{0}$. Assume that the policy is a participating one, or "with profit", in which the benefit $C_{T}$ that will be paid by the insurer if the policyholder is alive at time $T$ is determined by incrementing each year the sum insured by a fraction $\beta$ of the interest earned by the insurer on the investment of the premium ${ }^{4}$. The entire technical reserve is invested in a properly defined reference fund, which is directly managed by the insurance company.

Let $F_{t}$ be the market value at time $t$ of the fund where the premium is invested. The rate of return earned by the fund in year $[t-1, t]$ is:

$$
I_{t}=\frac{F_{t}}{F_{t-1}}-1
$$

obviously $I_{t}$ is a random variable which can also assume negative values. Let us assume that the basic actuarial calculations are performed at the technical interest rate $i$. The Italian system for profit sharing policies then provides that if $I_{t}>i$ a portion of the extra earned interest is credited to the insured by an increase of the sum insured. A typical interest crediting mechanism is obtained by readjusting the benefit at the end of the year $t$ with the rule:

$$
C_{t}=C_{t-1}\left(1+\rho_{t}\right), \quad t=1,2, \ldots, T,
$$

where the readjustment rate is defined as:

$$
\rho_{t}:=\frac{\max \left\{\beta I_{t}, i\right\}-i}{1+i}
$$

here $\beta \in(0,1)$ is the so called "participation coefficient". Both the technical rate $i$ and the $\beta$ coefficient are contractually specified, that is fixed at time zero.

The quantity $\beta I_{t}$ in expression (3) represents the portion of the fund return which is credited to the policyholder (by increasing the sum assured); the remaining portion $(1-\beta) I_{t}$ is retained by the insurer and determines his investment gain. The floor rate $i$ guarantees to the policyholder that the sum insured cannot decrease; thus the insurer must guarantee the readjusted benefit $C_{t}$ even if the reference fund does not realize the technical rate $i$ in year $t^{5}$.

Under this profit sharing rule, the final benefit is given by:

$$
C_{T}=C_{0} \Phi_{T}
$$

4 We refer to the case of a participating policy since this seems to provide the most challenging application of the NUMAT method. For unit-linked and index-linked policies the market based valuation approach appears to be natural (and probably unavoidable). A detailed example of NUMAT valuation of a participating whole life insurance contract can be found in Pacati (2003).

5 Similar contracts have been analyzed by several authors. Persson-Aase (1997) and Miltersen-Persson (1999), e.g. derive the price of minimum return guarantee under various interest rate models. GrosenJørgensen (2000), and Miltersen-Persson (2003) are particularly concerned with the effects of the return distribution mechanism on the price of the policy. A general framework for valuing return distribution and bonus policies under a stochastic "second order" basis has been proposed by Norberg (1999). 
where the readjustment factor $\Phi_{T}$ is defined as:

$$
\Phi_{T}:=\prod_{t=1}^{T}\left(1+\rho_{t}\right)=(1+i)^{-T} \prod_{t=1}^{T}\left(1+\max \left\{\beta I_{t}, i\right\}\right) .
$$

Let $\mathcal{E}(x, T)$ denote the event "the life insured aged $x$ is alive at time $T$ ", and let $\mathbb{\Xi}_{\mathcal{E}(x, T)}$ denote the indicator function of $\mathcal{E}(x, T)$. The liability $Y_{T}$ of the insurer at time $T$ is given by:

$$
Y_{T}=C_{0} \Phi_{T} \rrbracket_{\mathcal{E}(x, T)}
$$

hence the random payoff $Y_{T}$ is affected by both financial and "technical" (actuarial) uncertainty.

For this simple policy, if surrendering is not allowed the technical uncertainty is represented by survival risk. Financial uncertainty can be of a more complex nature, depending on the financial risk factors affecting the investment results. Given that the asset allocation of the typical reference funds is usually characterized by an important bond component, the interest rate risk will be of primary concern in the valuation framework.

\subsection{Linear valuation}

We assume that all the random variables concerning the valuation problem are defined on a probability space $(\Omega, \mathcal{A}, P)$, where $P$ is the probability measure we assume to describe all random movements in time (natural probability measure). In order to model financial and technical uncertainty, we also assume that two sub- $\sigma$-algebras can be defined: $\mathcal{F}$, containing the financial events, and $\mathcal{T}$, which contains the technical events. Correspondingly, we also consider the two filtrations:

$$
\mathcal{F}_{0} \subset \mathcal{F}_{1} \subset \cdots \subset \mathcal{F}_{t} \subset \cdots \subset \mathcal{F} ; \quad \mathcal{T}_{0} \subset \mathcal{T}_{1} \subset \cdots \subset \mathcal{T}_{t} \subset \cdots \subset \mathcal{T} .
$$

By definition, $\Phi_{T}$ is $\mathcal{F}_{T}$-measurable and $\mathbb{\Xi}_{\mathcal{E}(x, T)}$ is $\mathcal{T}_{T}$-measurable.

We are interested in assigning a value at time $t$ to the random variable $Y_{T}$ payable at time $T$. We call this map the Valuation Functional, denoted by $V\left(t ; Y_{T}\right)$. Again it suffices here to explain our procedure, if we show how to find $V\left(0 ; Y_{T}\right)$ (valuation at the beginning of the policy).

The functional $V\left(0 ; Y_{T}\right)$ should avoid riskless arbitrage opportunities; hence it must have the following representation:

$$
V\left(0 ; Y_{T}\right)=\mathbf{E}^{P}\left[\pi_{T} Y_{T}\right],
$$

where $\mathbf{E}^{P}$ is the conditional expectation, given $\mathcal{A}_{0}$, under the natural measure $P$ and $\pi_{T}$ (the "state-price deflator") is a strictly positive $\mathcal{A}_{T}$-measurable random 
variable 6 . This result is a central one in the theory of arbitrage pricing (see e.g. Duffie (1992)). It can also be derived by the Riesz representation theorem if the linearity of the Valuation Functional $V$ is required (see also Bühlmann (1995)).

We assume independence between financial and technical uncertainty; more precisely, we assume that $\mathcal{F}$ and $\mathcal{T}$ are independent and that the deflator $\pi_{T}$ can be expressed as the product of two strictly positive random variables:

$$
\pi_{T}=\varphi_{T} \chi_{T}
$$

where $\varphi_{T}$, the financial deflator, is $\mathcal{F}_{T}$-measurable and $\chi_{T}$, the technical deflator, is $\mathcal{T}_{T}$-measurable ${ }^{7}$. Under this assumption the following factorization holds:

$$
V\left(0 ; Y_{T}\right)=C_{0} \mathbf{E}^{P}\left[\varphi_{T} \Phi_{T}\right] \mathbf{E}^{P}\left[\chi_{T} \rrbracket_{\mathcal{E}(x, T)}\right] .
$$

\subsection{Financial component}

We can interpret the readjustment factor $\Phi_{T}$ as the stochastic payoff of an indexed Zero-Coupon Bond (ZCB), called $U$, maturing at time $T$. The reference index of $U$ is the annual return $I_{t}$ of the investment fund and the indexation rule is specified by the definition (4). By the separation property (8) the value of this indexed $\mathrm{ZCB}$ at time zero is equal to:

$$
u(0, T):=V\left(0 ; \Phi_{T}\right)=\mathbf{E}^{P}\left[\varphi_{T} \Phi_{T}\right] .
$$

It is important to distinguish (9) from a usual non-indexed ZCB with deterministic payoff $\mathbf{1}_{T}$ (one unit of cash at time $T$ ), for which we have:

$$
v(0, T):=V\left(0 ; \mathbf{1}_{T}\right)=\mathbf{E}^{P}\left[\varphi_{T}\right] .
$$

Assuming the financial market to be complete the payoff $\Phi_{T}$ (and of course also $\left.\mathbf{1}_{T}\right)$ can be replicated by a suitable trading strategy and the price $u(0, T)$ must be equal to the market price of this strategy, independently of the probability assessment and the risk aversion of the insurer. It is natural to suppose that short-term riskless (i.e. not defaultable) borrowing is allowed in the market

${ }_{6}$ If $f_{T}(y)$ is the probability density function of $Y_{T}$, we can also write:

$$
V\left(0 ; Y_{T}\right)=\int_{-\infty}^{\infty} \pi_{T}(y) Y_{T}(y) f_{T}(y) d y=\int_{-\infty}^{\infty} Y_{T}(y) \psi_{T}(y) d y,
$$

where $\psi_{T}(y):=\pi_{T}(y) f_{T}(y)$ is the so-called state-price density.

7 In more general situations the policy can be also surrendered. In this case the independence assumption in general is too strong, since the option to early redeem the contract can be exercised by the policyholder based on financial market events. However, for policies where early redemption is strongly penalized it could be never optimal to exercise the surrender option. In these cases redemptions seems to be essentially driven by the evolution of personal consumption plans and one can retain the independence assumption, modelling surrenders as "technical" events, described by experience-based elimination tables. For the application of results in American option pricing theory to the early exercise of interest rate guarantees see Grosen-Jørgensen (1997). 
at a strictly positive discount. Let $\delta_{T}$ denote the payback at time $T$ of a unit of money rolled over in short-term borrowing from 0 to $T$. It is well-known that in this case there exists a unique equivalent probability measure $Q$ such that:

$$
\mathbf{E}^{P}\left[\varphi_{T} \Phi_{T}\right]=\mathbf{E}^{Q}\left[\xi_{T} \Phi_{T}\right],
$$

where $\xi_{T}:=1 / \delta_{T}$ and $\mathbf{E}^{Q}$ is the expectation taken with respect to the $Q$ measure. In a continuous-time setting one has:

$$
\xi_{T}=e^{-\int_{0}^{T} r_{t} d t}
$$

where $r_{t}$ is the stochastic force of interest (the so-called "spot rate") prevailing on the market at time $t$ for riskless investments. The $Q$ measure is also referred to as the risk-neutral measure, since the price $V\left(0 ; \Phi_{T}\right)$ can be expressed as the expectation, under $Q$, of the terminal payoff $\Phi_{T}$ discounted with the stochastic discount factor $\xi_{T}$. In other words, one can "neutralize" the risk premia contained in the deflator $\varphi_{T}$ using the $Q$ measure instead of the natural measure $P$.

\subsection{Technical component}

For the technical component of the valuation problem, we look at:

$$
V\left(0 ; \rrbracket_{\mathcal{E}(x, T)}\right):=\mathbf{E}^{P}\left[\chi_{T} \rrbracket_{\mathfrak{E}(x, T)}\right],
$$

as the time zero value of $\rrbracket_{\mathcal{E}(x, T)}$. It is natural to assume:

$$
V\left(0 ; \mathbf{1}_{T}\right)=1,
$$

which implicitly states that no discounting effect is involved in this valuation, the "price of time" being completely taken into account by the financial component of the pricing procedure ${ }^{8}$. A crucial point now is that usually the technical "payoff" $\square_{\mathfrak{E}(x, T)}$ cannot be replicated by traded contracts. Hence the complete market assumption is not fulfilled and the price $V\left(0 ; \rrbracket_{\mathcal{E}(x, T)}\right)$ cannot be determined by observing the market. However, since the assumption (14) implies the norming condition $\mathbf{E}^{P}\left[\chi_{T}\right]=1$, we can write:

$$
V\left(0 ; \rrbracket_{\mathcal{E}(x, T)}\right)=\mathbf{E}^{D}\left[\rrbracket_{\mathcal{E}(x, T)}\right],
$$

8 In a more formal setting we could define two different valuation functionals:

$$
V^{F}\left(0 ; \Phi_{T}\right):=\mathbf{E}^{P}\left[\varphi_{T} \Phi_{T}\right], \quad V^{T}\left(0 ; \rrbracket_{\mathcal{E}(x, T)}\right):=\mathbf{E}^{P}\left[\chi_{T} \rrbracket_{\mathcal{E}(x, T)}\right],
$$

and express the factorization property (8) as:

$$
V\left(0 ; Y_{T}\right)=C_{0} V^{F}\left(0 ; \Phi_{T}\right) V^{T}\left(0 ; \rrbracket_{\mathcal{E}(x, T)}\right) .
$$

In this framework it would be natural to consider the discounting effect to be taken into account in the $V^{F}$ component by definition. Hence condition (14), which should be written as $V^{T}\left(0 ; \square_{\Omega}\right)=1$, would follow as a consequence of the no-arbitrage assumption. 
where $D$ is a transformed probability measure defined by $d D=\chi_{T} d P$. The use of an "adjusted" probability measure to preserve price additivity was suggested by Venter (1991). The idea has been developed by Wang $(1996,2000,2002)$ who proposed a number of "distortion" functions of the $P$ measure providing desirable properties of the price functional.

Once the $D$ measure has been chosen by the insurer, the quantity:

$$
\overline{\bar{C}}_{0}:=C_{0} \mathbf{E}^{D}\left[\mathbb{1}_{\mathcal{E}(x, T)}\right]
$$

can be considered as a definition of the certainty equivalent, at time $T$, of the random amount $C_{0} \rrbracket_{\mathcal{E}(x, T)}$. As the $P$ measure represents the natural probability assessment of the insurer, the difference:

$$
C_{0}\left\{\mathbf{E}^{D}\left[\mathbb{q}_{\mathcal{E}(x, T)}\right]-\mathbf{E}^{P}\left[\mathbb{1}_{\mathfrak{E}(x, T)}\right]\right\}
$$

expresses the risk loading (at time $T$ ) required for bearing the survival risk. For the relations between this approach and the expected utility theory see Wang (1996).

Even if the reinsurance markets can not be considered as being purely competitive, some useful indications for a market based assessment of the $D$ measure, and hence for the price $V\left(0 ; \square_{\mathfrak{E}(x, T)}\right)$, could be obtained by observing the current prices of reinsurance treaties for survival/mortality risk. However, the issue of how in general a market consensus on the value of technical payoffs can be determined is currently an open problem. In the sequel we shall assume the $D$ measure simply as given.

\subsection{The numeraire}

Under this set of assumptions, the time zero price of the random benefit $Y_{T}$ can be given as:

$$
V\left(0 ; Y_{T}\right)=C_{0} \mathbf{E}^{Q}\left[e^{-\int_{0}^{T} r_{t} d t} \Phi_{T}\right] \mathbf{E}^{D}\left[\mathbb{Q}_{\mathcal{E}(x, T)}\right]
$$

The technical expectation can be expressed by the usual actuarial notation, that is:

$$
\mathbf{E}^{D}\left[\mathbb{q}_{\mathcal{E}(x, T)}\right]={ }_{T} p_{x},
$$

where the probability ${ }_{T} p_{x}$ is computed using suitable mortality tables. The riskneutral expectation provides the price of the indexed ZCB $U$ :

$$
u(0, T)=\mathbf{E}^{Q}\left[e^{-\int_{0}^{T} r_{t} d t} \Phi_{T}\right] .
$$

Then the expression (16) can be written as:

$$
V\left(0 ; Y_{T}\right)=C_{0} u(0, T)_{T} p_{x} .
$$


The structure of this expression is similar to the expression of the technical reserve $R_{0}$ of the policy at time zero; in the traditional framework we have:

$$
R_{0}=C_{0}(1+i)^{-T} p_{x}^{\prime},
$$

where $i$ is the technical rate and ${ }_{T} p_{x}^{\prime}$ is computed using "first order" mortality tables. It is well-known that first order tables are usually based on conservative assumptions, hence ${ }_{T} p_{x}^{\prime}$ is a "distorted" probability providing an implicit safety loading. To simplify the exposition we shall assume that the first order probabilities are the same as those given by the $D$ measure; i.e. we pose:

$$
{ }_{T} p_{x}^{\prime}={ }_{T} p_{x} .
$$

Even with this assumption it is clear that (19) and (20) represent very different valuation approaches; in particular, $u(0, T)$ can be interpreted as a market based discount factor, as opposed to the contractual discount factor $(1+i)^{-T}$.

The expression (19) makes also clear that the financial component of this valuation approach is - using Bühlmann's terminology - a numeraire approach; we can consider the benefit $Y_{T}$ provided by the participating pure endowment policy as expressed in "units" of the indexed ZCB $U$; the current price of the benefit is given by $\bar{C}_{0}:=C_{0 T} p_{x}$ times the current market price $u(0, T)$ of the unit.

One can also define:

$$
V^{*}\left(0 ; Y_{T}\right):=\frac{V\left(0 ; Y_{T}\right)}{u(0, T)}
$$

where $V^{*}=\bar{C}_{0}$ is the relative price of $Y_{T}$ with respect to $U$, that is the price of $Y_{T}$ measured in units of $U$ rather than in Euros. In financial economics, expressing the price by $V^{*}$ instead of $V$ is usually referred to as a "change of numeraire".

As a particular case, one can consider a non participating policy, where $C_{T}=C_{0}$ (i.e. $\Phi_{T}=\mathbf{1}_{T}$ ); now one has:

$$
V\left(0 ; Y_{T}\right)=C_{0} v(0, T)_{T} p_{x},
$$

where:

$$
v(0, T):=\mathbf{E}^{Q}\left[e^{-\int_{0}^{T} r_{t} d t}\right]
$$

is the risk free discount factor prevailing on the financial market at time zero for the maturity $T$; that is the current price of the default free unit discount bond maturing at time $T\left(B_{0}(T)\right.$ in the Aase-Persson (2003) notation).

\section{FAIR VALUATION, VBIF AND EMBEDDED OPTIONS}

If we are able to determine the market value $u(0, T)$ of the unit, expression (19) provides a market based valuation of the outstanding liabilities generated by 
the policy, what is usually said a fair valuation ${ }^{9}$. Since $u(0, T)$ must be typically derived using an appropriate stochastic pricing model, we usually refer to $V_{0}:=V\left(0 ; Y_{T}\right)$ as the stochastic reserve, as opposed to the traditional reserve $R_{0}$ given by (20).

\subsection{Value of Business in Force: the stochastic reserve approach}

Under the assumption of complete financial market, the payoff $C_{T}$ can be replicated by a trading strategy and the amount $C_{0} u(0, T)$ represents the market price at time zero of the equivalent portfolio, that is of the the dynamic portfolio of traded securities which replicates the stochastic benefit $C_{T}$. Therefore, assuming that the mortality risk can be "diversified away" in a large portfolio, the stochastic reserve $V_{0}$ represents the market price at time zero of the portfolio which replicates the stochastic liability $Y_{T}$ of the insurer. It is then natural to define the time zero value $E_{0}$ of the business in force (the so called VBIF, net of expenses, administrative costs and taxes), as the difference:

$$
E_{0}:=R_{0}-V_{0}
$$

The interpretation of this expression is straightforward. The technical reserve $R_{0}$ is the capital required at time zero to the insurer in order to issue the policy ${ }^{10}$, while $V_{0}$ is the investment actually needed at time zero to meet the future liabilities. The difference $E_{0}=R_{0}-V_{0}$, which usually should be positive, is not immediately available to the insurer, but will be progressively delivered in the future as profits emerging during the life of the policy; however the present value of these profits, by arbitrage, must be equal to $E_{0}$.

This method for determining $E_{0}$ can be called the "stochastic reserve approach". Usual decompositions of VBIF, e.g. separation between investment component and mortality component, can be recovered under this method by using different specifications of the probability ${ }_{T} p_{x}$ in (19) and of the probability ${ }_{T} p_{x}^{\prime}$ in (20). We considered here VBIF at time zero only for illustration; all the definitions can be immediately extended at any time $t$ during the life of the policy, as illustrated in section (5.4).

9 The International Accounting Standards Committee (IASC) in the Insurance Issues Paper (November 1999) defines the fair value as: "The amount for which an asset could be exchanged, or a liability settled, between knowledgeable, willing parties in an arm's-length transaction." A similar definition is adopted by the Financial Accounting Standard Board (FASB) in a Preliminary Views document (December, 1999): "Fair value is an estimate of the price an entity would have realized if it had sold an asset or paid if it had been relieved of a liability on the reporting date in an arm's-length exchange motivated by normal business considerations. That is, it is an estimate of an exit price determined by market interactions." By acknowledging these positions, the CAS Fair Value Task Force (CAS Task Force, 2000) defined fair value as: "the market value, if a sufficiently active market exists, OR an estimated market value, otherwise."

${ }^{10}$ We do not consider here the capital requirements given by the regulatory solvency margin. 


\subsection{The annual profits approach}

The standard method for measuring VBIF is an "annual profits approach". The method is based on typical recursive relations for the technical reserve which allows to define an annual sequence of expected technical gains $\bar{G}_{t}$ during all the life of the policy (a typical expression of this is known as the Homans formula). The cash-flow $\bar{G}_{t}$ can be considered as the expected annual profit to the insurer provided by the policy at the end of year $t$ and the VBIF is obtained as the present value of this cash-flow stream, derived under "Risk Adjusted Discounting" (RAD), that is by using a risk adjusted discount rate appropriate to the riskiness of the cash-flows. It is also usual a decomposition of VBIF based on the definition of investment gains and mortality gains.

It can be easily proven (De Felice-Moriconi (2002b, p. 94)) that under the no-arbitrage assumption in perfect markets the annual profits approach is equivalent to the stochastic reserve approach, provided that the traditional method is applied in a market based framework. This means that the expected annual gains must be derived under the risk-neutral measure $Q$ and then discounted at the risk free market rate. As we know, this approach correctly takes into account risk premia, and avoids arbitrage opportunities if the value $E_{0}$ is used as a market price. In principle, the RAD method is not inconsistent with the no-arbitrage approach; the Capital Asset Pricing Model, for example, prescribes - once the riskiness of the cash flow to be discounted has been correctly measured -, the correct risk adjusted discount rate which provides a market based, and arbitrage-free, valuation. However, in the applications the RAD approach suffers of the high degree of subjectivity involved in the practical assessment of both the expected cash-flows and the risk adjusted rate ${ }^{11}$.

One can have even more important problems with the RAD method when option-like payoffs are considered, as it happens in our life insurance applications. Usually the RAD method is applied as a single-scenario method; that is a single assumption is made on the future path of the returns from the investment of the reserve. This scenario of returns can be interpreted as the expected evolution of future return; the expected investment gains are determined by inputing these returns into the recursive equation. In typical situations the options embedded into the life insurance policies are out-of-the-money, that is the minimum guaranteed annual return (usually the technical interest rate $i$ ) is below the level of the assumed scenario returns; therefore the embedded options never goes in-the-money and the cost of the minimum annual guarantees is zero.

11 This difficulty is explicitly recognized by many scholars. Referring to the problem of properly specifying the exact evolution over time of the discounted expected value representation, Dybvig-Ross (1987, p. 104) argue that "This difficulty is usually overlooked in capital budgeting applications, which is probably not so bad, given the imprecision of our estimates of risk premia and future cash flows". Le Roy (1987, p. 948) observes that when the net present value principle is applied in incomplete markets under uncertainty "even if all agents agree about the probability distribution of the returns on a project, unanimity may break down". A comparison between a risk adjusted discount approach and an option-like approach to the pricing and reserving of maturity guarantees embedded in some life insurance policies has been made by Boyle-Hardy (1997). Their numerical results suggest that the consistency between the two methods is still far to be settled. 
A comparison between VBIF computed on a sample portfolio by the stochastic reserve approach and by the RAD method under scenario is provided in De Felice-Moriconi (2002b, p. 106).

\subsection{The embedded options}

In order to measure the cost of the minimum guarantees embedded in the insurance contract, one can compare the value of the policy with the "base value" of the policy, that is the value of an analogous policy without any guarantee. Let us define the "base readjustment rate" as:

$$
\rho_{t}^{B}:=\frac{\beta I_{t}-1}{1+i}
$$

and the "base readjustment factor" as:

$$
\Phi_{T}^{B}:=\prod_{t=1}^{T}\left(1+\rho_{t}^{B}\right)=(1=i)^{-T} \prod_{t=1}^{T}\left(1+\beta I_{t}\right) .
$$

Obviously $\Phi_{T}^{B} \leq \Phi_{T}$.

Under the market based approach, the "base value" of the contract is given by:

$$
B_{0}:=C_{0} V\left(0 ; \Phi_{T}^{B}\right)_{T} p_{x}
$$

where:

$$
V\left(0 ; \Phi_{T}^{B}\right)=\mathbf{E}^{Q}\left[e^{-\int_{0}^{T} r_{t} d t} \Phi_{T}^{B}\right]
$$

Thus the value of the guarantee can be defined as:

$$
O_{0}^{P}:=V_{0}-B_{0}=C_{0}\left[V\left(0 ; \Phi_{T}\right)-V\left(0 ; \Phi_{T}^{B}\right)\right]_{T} p_{x} .
$$

The $O_{0}^{P}$ value can be defined as the price of a put option because a contract with payoff $\left[\Phi_{T}-\Phi_{T}^{B}\right]^{+}=\Phi_{T}-\Phi_{T}^{B}$ gives the right to the holder to exchange the result $\Phi_{T}^{B}$ of an unguaranteed contract with the result of the guaranteed one if the return $I_{t}$ realized by the reference fund will not be greater than $i / \beta$ over each year of the contract. Given that the minimum return $i$ is guaranteed in each year and is "consolidated" for the successive years, this kind of option is a "ratchet-type" (or "cliquet") option. Of course, the price $O_{0}^{P}$ of this option is usually much greater than the price of a corresponding "maturity option", that is an option with (unit) minimum guaranteed amount $(1+i)^{T}$ at time $T$ (for some numerical examples, see De Felice-Moriconi (2002b, pp. 61-62)).

The expression:

$$
V_{0}=B_{0}+O_{0}^{P}
$$


can be referred to as the put decomposition of the contract. One can also define the "call decomposition" as:

$$
V_{0}=G_{0}+O_{0}^{C},
$$

where $G_{0}:=C_{0} v(0, T)_{T} p_{x}$ is the value of the minimum guaranteed terminal benefit (which is obtained for $\Phi=1$ ), and:

$$
O_{0}^{C}:=V_{0}-G_{0}=C_{0}[u(0, T)-v(0, T)]_{T} p_{x}
$$

represents the price of the ratchet call option giving to the holder the right to participate to the investment returns if the result will be greater than the minimum guaranteed result. We observe that in Bühlmann's terminology, $G_{0}$ is the value of the VaPo (see Bühlmann, 2003b, Baumgartner-Bühlmann-Koller, 2004).

\section{Determining the VAlUATION FACTOR OR "PRICING THE UNIT"}

\subsection{Extracting information from the market}

The central problem under the NUMAT approach is the pricing of the unit $U$, that is the market based determination of the price $u(0, T)=V\left(0 ; \Phi_{T}\right)$; since:

$$
V\left(0 ; C_{T}\right)=C_{0} u(0, T),
$$

$u(0, T)$ represents the appropriate valuation factor for the random benefit $\mathrm{C}_{T}=$ $\mathrm{C}_{0} \Phi_{T}$.

We observed that for non participating policies, that is when $\Phi_{T}=1$, the valuation factor reduces to $v(0, T)$, that is it is equal to the current price of the default free unit ZCB with maturity $T$. In this case the determination of the market price does not pose any serious problem. In fact the determination of $v(0, T)$ for maturity $T \leq 30$ years is an ordinary job for the financial operators. Currently swap rates $w_{T}$ for maturity up to 30 years are daily quoted on the interest rate swap market (e.g. Euribor); as it is well-known, $w_{T}$ represents the $T$-years par-yield, that is the level of the annual coupon of a unit straight bond which is quoted at par (i.e. has price 1):

$$
w_{T}=\frac{1-v(0, T)}{\sum_{t=1}^{T} v(0, t)} .
$$

Therefore, if one can observe the current values of $w_{T}$ for $T=1,2, \ldots, 30$, one can immediately derive the zero-coupon term structure $v(0, T)$ for $T=1,2, \ldots, 30^{12}$. The method is referred to as a "bootstrapping procedure", but it consists in nothing else than solving the linear system:

${ }^{12}$ The maturities effectively quoted are $T=1$ to $10,12,15,20,25,30$; usually the annual sequence of the swap rates is completed by some kind of interpolation. 


$$
w_{T} \sum_{t=1}^{T-1} v(0, t)+\left(1+w_{T}\right) v(0, T)=1, \quad T=1,2, \ldots, 30
$$

In the trivial case of $\Phi_{T}=1$ we can directly extract from the market the information needed for the valuation, and the explicit determination of the riskneutral measure $Q$ in (24) is not required.

Things are not so simple for a participating policy since, given the complexity of the interest crediting mechanism given by (2) and (3), the readjustment factor $\Phi_{T}$ represents the payoff of a security, the unit, which is not effectively traded. However, for the complete market assumption, this unit is a redundant security and can be priced via equation (18) using a suitable stochastic model calibrated on the market data.

To illustrate this crucial point, let us assume for the moment that the reference fund $F$ underlying the policy is composed only of interest rate sensitive contracts (e.g. government bonds), so that the factor $\Phi_{T}$ is affected only by interest rate risk. In this case a stochastic model for interest rates is sufficient for pricing the unit. For simplicity sake, assume a typical one-factor Markov model having the spot rate $r_{t}$ as the state variable; hence, a complete identification of the model will require the specification of a parameter vector $\mathbf{p}$ determining the conditional probability distribution of the spot rate process, and a parameter vector $\mathbf{r}$ representing the structure of the risk premia (the "term premia") prevailing on the interest rate market. Clearly, the parameters $\mathbf{p}$ determine the (financial component of) the probability measure $P$; however the riskneutral valuation rule (18) prescribes the prices as being determined only by the risk adjusted measure $Q$. This probability measure will be identified by a new parameter vector $\hat{\mathbf{p}}$ whose components will be some function of the components of $\mathbf{p}$ and $\mathbf{r}$. In general the knowledge of $\mathbf{p}$ and $\mathbf{r}$ separately cannot be inferred from the knowledge of the risk adjusted parameter vector $\hat{\mathbf{p}}$; however only $\hat{\mathbf{p}}$ is needed for pricing purposes.

Suppose that the model provides an explicit formula for the price of an interest rate sensitive security, e.g. for the price $v(0, T)$ of the unit ZCB; for a fixed maturity $T$ the bond price will be a function only of the risk adjusted parameters $\hat{\mathbf{p}}$ :

$$
v(0, T)=v(0, T ; \hat{\mathbf{p}}) .
$$

Thus we can identify the risk-neutral measure $Q$ by a calibration procedure consisting in estimating the parameters $\hat{\mathbf{p}}$ which provide a best fitting between model prices and observed prices. For example we can extract the information contained in the current structure of the interest rate swap $w_{T}$ by minimizing over the set of the possible values for $\hat{\mathbf{p}}$ the sum of squared errors:

$$
\Sigma^{2}:=\sum_{T=1}^{30}\left[W_{T}(\hat{\mathbf{p}})-1\right]^{2}
$$

where: 


$$
W_{T}(\hat{\mathbf{p}}):=w_{T} \sum_{t=1}^{T-1} v(0, t ; \hat{\mathbf{p}})+\left(1+w_{T}\right) v(0, T ; \hat{\mathbf{p}})
$$

is the model price of a $T$-years unit straight bond with coupons equal to $w_{T}$.

\subsection{Specification of the valuation model}

Typically the reference funds backing life insurance liabilities contain both bonds and stocks (at least); thus in order to model properly the yearly returns $I_{t}$ which determine the readjustment of the contractual benefits we have to model both interest rate and stock market risk ${ }^{13}$.

In many applications we adopt a two-factor diffusion model obtained by joining a one-factor Cox-Ingersoll-Ross (CIR) model for the interest rate risk and a Black-Scholes (BS) model for the stock market risk; the two sources of uncertainty are correlated. For liabilities providing also inflation protection a three-factor model, obtained by properly extending the CIR component in order to include both real and nominal interest rates (see Moriconi 1994, 1995), is employed.

\subsubsection{Interest rate uncertainty}

The single source of uncertainty is the spot rate $r_{t}$, which is a diffusion process described by the stochastic differential equation:

$$
d r_{t}=f^{r}\left(r_{t}, t\right) d t+g^{r}\left(r_{t}, t\right) d Z_{t}^{r},
$$

where $Z_{t}^{r}$ is a standard Brownian motion. In the CIR model the drift function is chosen as:

$$
f^{r}\left(r_{t}, t\right):=\alpha\left(\gamma-r_{t}\right), \quad \alpha, \gamma>0,
$$

and the diffusion function is defined by:

$$
g^{r}\left(r_{t}, t\right):=\rho \sqrt{r_{t}}, \quad \rho>0 .
$$

Thus it is assumed a mean-reverting drift, with long term rate $\gamma$ and speed of adjustment $\alpha$, and a "square root" diffusion, with volatility parameter $\rho$. As it is well-known, this "mean-reverting square-root" process implies a non-central chi-squared transition density for $r_{t}$ (Feller, 1951).

\footnotetext{
${ }^{13}$ As a general consistency rule, assets and liabilities must be valued under the same market model; thus the valuation model must have at least as many risk factors as are required to price the asset portfolio. Of course, additional factors are needed if the liabilities are also linked to some exogenous market index; for example, if the policy also provides some kind of inflation protection of benefits, the valuation model for the liabilities must include an additional source of uncertainty for the real interest rate risk, independently of inflation linked bonds are held in the reference fund.
} 
The Vasicek model (and its usual extensions) is more simple than the CIR model and is widely used for pricing interest rate derivatives. Since this model assumes a normal transition distribution, it assigns positive probability to negative values of the spot rate; for long maturities this can have relevant effects, producing discount factors greater than one. Therefore the Vasicek model appears inadequate to life insurance applications. The CIR model seems to offer a good trade-off between economic consistency and mathematical tractability ${ }^{14}$.

In the CIR model the preferences prevailing on the market (the market price of interest rate risk) are specified by the function:

$$
h^{r}\left(r_{t}, t\right):=\pi \frac{\sqrt{r_{t}}}{\rho}, \quad \pi \in \mathbb{R}
$$

hence the parameter vector for the risk premia is simply $\mathbf{r}=\{\pi\}$. Under the CIR approach - which is a general equilibrium approach - it is shown that this form of the preference function avoids riskless arbitrage.

\subsubsection{Stock price uncertainty}

Also for the stock market we assume a single source of uncertainty, expressed by the stock index $S_{t}$; the diffusion process for the stock index is given by the stochastic differential equation:

$$
d S_{t}=f^{S}\left(S_{t}, t\right) d t+g^{S}\left(S_{t}, t\right) d Z_{t}^{S},
$$

where $Z_{t}^{S}$ is a standard Brownian motion with the property:

$$
\operatorname{Cov}_{t}\left[d Z_{t}^{r}, d Z_{t}^{S}\right]=\eta d t, \quad \eta \in \mathbb{R} .
$$

Since we assume a BS-type model, we specify $f^{S}$ and $g^{S}$ as:

$$
f^{S}\left(S_{t}, t\right):=\mu S_{t}, \quad \mu \in \mathbb{R},
$$

and:

$$
g^{S}\left(S_{t}, t\right):=\sigma S_{t}, \quad \sigma>0 .
$$

Thus we have a geometric Brownian motion, with instantaneous expected return $\mu$ and volatility $\sigma$, which implies a lognormal transition density for $S_{t}$.

To prevent arbitrage, the market price of risk for the stock market has the classical form:

$$
h^{S}\left(S_{t}, t\right):=\frac{\mu-r_{t}}{\sigma}
$$

\footnotetext{
${ }^{14}$ For a valuation of interest rate guarantees with the Heath-Jarrow-Morton model see MiltersenPersson (1999).
} 
thus no additional parameter is needed in order to specify the preferences in this case $^{15}$.

\subsubsection{The valuation equation}

By the Markov property, the time $t$ price of any security is a function of the state variables; in particular, for the unit price we have:

$$
u(t, T)=u\left(r_{t}, S_{t}, t ; T\right), \quad 0 \leq t \leq T .
$$

Under the usual perfect market conditions the no-arbitrage principle, via the hedging argument, leads to the general valuation equation:

$$
\begin{aligned}
& \frac{1}{2}\left(g^{r}\right) \frac{\partial^{2} u}{\partial r^{2}}+\frac{1}{2}\left(g^{S}\right)^{2} \frac{\partial^{2} u}{\partial S^{2}}+\eta g^{r} g^{S} \frac{\partial^{2} u}{\partial r \partial S} \\
& +\left(f^{r}+g^{r} h^{r}\right) \frac{\partial u}{\partial r}+\left(f^{S}-g^{S} h^{S}\right) \frac{\partial u}{\partial S}+\frac{\partial u}{\partial t}=r u .
\end{aligned}
$$

This equation must be solved under the appropriate boundary conditions, including, for the unit price, the terminal condition:

$$
u(T, T)=\Phi_{T}
$$

As it is well-known, the no-arbitrage assumption requires the existence of an equivalent martingale measure $Q$ such that the discounted price process:

$$
u(t, T) e^{-\int_{0}^{t} r_{\tau} d \tau}, \quad 0 \leq t \leq T
$$

is a martingale with respect to $Q$. Under this martingale property the solution of the valuation equation under condition (40) has the integral expression:

$$
u(t, T):=V\left(t ; \Phi_{T}\right)=\mathbf{E}_{t}^{Q}\left[e^{-\int_{t}^{T} r_{z} d z} \Phi_{T}\right]
$$

also known as Feynman-Kac representation. For $t=0$ this gives expression (18).

\subsubsection{Identifying the risk-neutral measure}

By our choice of the functions $f^{r}, g^{r}, f^{S}$ and $g^{S}$ and of the correlation between the sources of uncertainty, the parameter vector related to the $P$ measure is:

$$
\mathbf{p}=\{\alpha, \gamma, \rho, \mu, \sigma, \eta\},
$$

\footnotetext{
15 In the usual formulations of the BS model no assumption on the risk premia is made at this stage, since relation (37) will be obtained as a consequence of the hedging argument which leads to the valuation equation. For the sake of exposition we prefer to state this property here.
} 
while the parameter vector for the risk premia is simply $\mathbf{r}=\{\pi\}$. By inspection of the valuation equation (39) one observes that the coefficients of the first order derivatives with respect to $r$ and $S$ are not expressed by the "natural" drift functions $f^{r}$ and $f^{S}$, but are given by the modified functions:

$$
\hat{f}^{r}:=f^{r}+h^{r} g^{r}=\alpha\left(\gamma-r_{t}\right)+\pi r_{t}=\alpha \gamma-(\alpha-\pi) r_{t},
$$

and:

$$
\hat{f}^{S}:=f^{S}-h^{S} g^{S}=r_{t} S_{t} .
$$

These are the risk adjusted drifts ${ }^{16}$ which determine the form of the risk-neutral measure $Q$; the risk adjusted parameter vector is given by:

$$
\hat{\mathbf{p}}=\{\hat{\alpha}, \hat{\gamma}, \rho, \sigma, \eta\},
$$

where $\hat{\alpha}:=\alpha-\pi$ and $\hat{\gamma}:=\alpha \gamma$. Any solution of the valuation equation will be a function of this set of parameters and any calibration of the model to the observed prices will be performed by optimally choosing these parameters. The estimation of the effective mean reverting parameters for $r_{t}$ and the instantaneous expected return for $S_{t}$ is not needed for pricing purposes, since their value has no effect on the prices.

\subsubsection{Measures of financial risk}

Since the price $u(t, T)$ is a function of the state variable $r_{t}$ and $S_{t}$, it is natural to express the risk inherent to these sources of uncertainty as a sensitivity measure. For the interest rate risk it is usual to define:

$$
\Omega^{r}\left(\Phi_{T}\right):=-\frac{\partial u(0, T)}{u(0, T) \partial r}
$$

for the particular case of the deterministic unit ZCB we define:

$$
\omega(T):=-\frac{\partial v(0, T)}{v(0, T) \partial r}
$$

The "stochastic duration" $D\left(\Phi_{T}\right)$ is defined as the maturity of the deterministic ZCB with the same risk of $\Phi_{T}$; hence:

$$
D\left(\Phi_{T}\right):=\omega^{-1}\left(\Omega^{r}\right) .
$$

\footnotetext{
${ }^{16}$ The valuation equation (39) can be easily recognized as the backward Kolmogoroff equation for the bivariate diffusion $\left\{r_{t}, S_{t}\right\}$ with drifts $\hat{f}^{r}$ and $\hat{f}^{S}$, with diffusion coefficients $g^{r}$ and $g^{r}$, correlation $\eta$ and "killing rate function" $r_{t}$ (see Karlin-Taylor (1981, pp. 222-224)).
} 
Because of the mean reversion effect the function $\omega(T)$ is bounded; hence for high values of $\Omega^{r}$, that is for contracts with strong interest rate risk, the stochastic duration $D\left(\Phi_{T}\right)$ could also not exist. Of course this is not a problem for controlling interest rate risk since one can directly use the sensitivity $\Omega^{r}$ as a measure of risk. In our applications the stochastic duration $D\left(\Phi_{T}\right)$ usually is well defined and is typically shorter than the maturity $T$ of the policy ${ }^{17}$.

The sensitivity of price to stock market index has a similar definition:

$$
\Omega^{S}\left(\Phi_{T}\right):=\frac{\partial u(0, T)}{u(0, T) \partial S} ;
$$

the derivative with respect to $S$ is well-known as the Delta of the contract:

$$
\text { Delta }:=\frac{\partial u(0, T)}{\partial S} \text {. }
$$

Of course, for $\Phi_{T}=1$ one has $\Omega^{S}=0$ since $v(0, T)$ is independent of $S$.

\subsubsection{Risk Based Capital}

The NUMAT approach also provides a straightforward methodology for determining levels of absorbed capital, which are risk measures currently used in strategic planning and in solvency rating problems. Consider a portfolio of contracts with stochastic reserve $V_{0}$ at time zero. For a given risk factor, the risk based capital, or economic capital, of the portfolio is the maximum unexpected loss in portfolio's value, in a time period $\theta$ with probability $\varepsilon$, caused by an adverse movement of the risk factor. While the definition is quite similar to the well-known Value-at-Risk (VaR) definition, here the values of the parameters $\theta$ and $\varepsilon$ are essentially different. Usually in risk capital definitions the time period $\theta$ is equal to 1 year (the usual accounting horizon), which is longer than the typical "unwinding periods". Moreover, since the risk capital can be interpreted as the adequate cushion for the insurance company to maintain a given credit rating, $\varepsilon$ is chosen as the typical figure of the one-year default probability corresponding to this rating; for companies interested in strong credit quality this probability can be very low.

Let us refer to a "portfolio" consisting only of our pure endowment policy with terminal benefit $C_{T}=C_{0} \Phi_{T}$; the portfolio value at time zero is:

$$
V_{0}:=C_{0} V\left(0 ; \Phi_{T}\right)=C_{0} u(0, T)_{T} p_{x} .
$$

\footnotetext{
${ }^{17}$ Usually it turns out that the interest rate sensitivity (and the stochastic duration) of a participating policy is considerably lower than the sensitivity of a corresponding non participating policy. This self-immunization property is essentially similar to the analogous property displayed by floating rate notes, having interest rate sensitivity similar to short term bonds, despite their mid/long maturity (De Felice-Moriconi-Salvemini (1993, pp. 137-138)). This is an important result since it suggests that the traditional duration mismatching between assets and liabilities in life insurance can be strongly reduced (in terms of sensitivity) for portfolios of participating policies. Values of the stochastic duration for an outstanding policy portfolio, as well as applications to asset-liability management are reported in De Felice-Moriconi (2002b, p. 86, pp. 90-91).
} 
As concerning the financial risk, under the two-factor model:

$$
u(0, T)=u\left(r_{0}, S_{0}, 0 ; T\right),
$$

and one has the risk capital $K^{r}$ induced by $r_{t}$ (the interest rate risk capital) and the risk capital $K^{S}$ induced by $S_{t}$ (the stock price risk capital). Since the valuation factor is usually monotonic both with respect to $r$ and $S$, the risk capitals $K^{r}$ and $K^{S}$ can be directly obtained from percentiles of the probability distributions of $r$ and $S$ ("underlying percentile method").

The function $u$ is usually monotonic decreasing with respect to $r$, hence interest rate risk capital can be defined as:

$$
K^{r}:=v(0, \theta)_{\theta} p_{x} C_{0}\left[u\left(r_{\theta}^{*}, \bar{S}_{\theta}, \theta ; T\right)-u\left(\bar{r}_{\theta}, \bar{S}_{\theta}, \theta ; T\right)\right] \mathbf{E}^{P}\left[{ }_{T-\theta} p_{x+\theta}\right],
$$

where $\bar{r}_{\theta}:=\mathbf{E}^{P}\left[r_{\theta}\right]$ and $\bar{S}_{\theta}:=\mathbf{E}^{P}\left[S_{\theta}\right]$ is the expected value at time zero of the spot rate and of the stock price, respectively, at the end of the period $\theta$, and $r_{\theta}^{*}$ is the "underlying percentile", defined by $\mathbf{P}\left(r_{\theta} \leq r_{\theta}^{*} \mid r_{0}\right)=\varepsilon$.

Similarly, since $u$ is monotonic increasing with respect to $S$, the stock market risk capital can be given by:

$$
K^{S}:=v(0, \theta)_{\theta} p_{x} C_{0}\left[u\left(\bar{r}_{\theta}, S_{\theta}^{*}, \theta ; T\right)-u\left(\bar{r}_{\theta}, \bar{S}_{\theta}, \theta ; T\right)\right] \mathbf{E}^{P}\left[{ }_{T-\theta} p_{x+\theta}\right],
$$

where $S_{\theta}^{*}$ is implicitly defined by $\mathbf{P}\left(S_{\theta} \leq S_{\theta}^{*} \mid S_{0}\right)=1-\varepsilon$.

It is worthwhile to observe that the probability measure to be used in these computations is the natural measure $P$; thus some amount of subjectivity is necessarily involved, since $P$ cannot be estimated on the market and some parameters of the distributions must be specified based on personal beliefs (or however using additional estimations). For the stock market component the parameter $\mu$ of the lognormal distribution for $S$ must be fixed; as concerning the interest rate component, the most straightforward way for determining the parameters of the non-central chisquared distribution of $r$ consists in fixing exogenously the value of the long term interest rate $\gamma$ and then deriving the mean-reversion coefficient as $\alpha=\hat{\gamma} / \gamma$.

Mortality risk capital $K^{M}$ can be derived by similar computations, based on a properly defined worst case for ${ }_{T} p_{x}$. This requires, of course, to specify a model for mortality risk; however, given our independence assumptions, the financial risk capitals $K^{r}$ and $K^{S}$ will not be affected by stressed mortality scenarios ${ }^{18}$.

\section{APPLYing THE VAlUation MODEL}

\subsection{Some calibration details}

The parameters $\hat{\alpha}, \hat{\gamma}$ and $\rho$ of the CIR component of the valuation model can be estimated by calibration on the market of the interest rate sensitive securities.

${ }^{18}$ Economic capitals for technical risks can be particularly high in non-life insurance, where the determination of the ultimate reserves and the premium rating are exposed to large estimation errors. For an application of the stochastic chain-ladder method to the determination of the reserve risk capital and of the premium risk capital in P\&C insurance see De Felice-Moriconi (2003). 
Since the CIR model provides an explicit formula for $v(t, T)$ (Cox-IngersollRoss (1985), p. 393), the calibration can be made on the current cross section of swap rates by performing the nonlinear regression (34) described in section 4.1. However under the CIR model it can happen that different sets of parameters are found having very similar levels of $\Sigma^{2}$ but different values for the volatility parameter $\rho$. While the use of these different parameter sets produces similar prices for linear products (that is for products which can be expressed as static portfolios of unit ZCBs), different values of $\rho$ can produce important discrepancies in the valuation of contracts with non linear payoff, as the options embedded in our life insurance liabilities. In order to overcome this difficulty, one can extend the set of market data by including also prices of interest rate options in the estimation procedure. In our applications we usually perform the calibration of the CIR model using both the swap rates and a set of quoted prices for interest rate caps and floors, that can be easily done given that explicit formulae for the price of these derivatives are available using the CIR model. The method usually produces values of $\rho$ which explain fairly well the observed option prices while maintaining a good fitting with the observed yield curve.

The remaining parameters $\sigma$ and $\eta$ have in some sense a more strategic nature and can be exogenously specified. Usually we assume for $\sigma$ the same value of the historical volatility of the stock component of the reference fund. For the correlation coefficient $\eta$ we adopt figures derived by classical econometric studies on the Italian market (a slightly negative value is usually assumed); however for typical values of the other parameters the value of $\eta$ seems to have a weak influence on the valuation procedure.

\subsection{Numerical computation}

Given the complexity of the profit sharing rule, the valuation factor $u(0, T)$ must be derived by the valuation equation using numerical methods. We usually compute the risk neutral expectation (18) using Monte Carlo simulations for the bivariate process $\left\{r_{t}, S_{t}\right\}$. Properly incrementing the starting values $r_{0}$ and $S_{0}$ of the Monte Carlo recursions we also obtain numerical derivatives of the price, which provide the relevant financial risk measures, and worst case valuations for risk capital computations ${ }^{19}$.

\subsection{Defining the underlying of the policy}

A critical point for determining the price $V_{0}$ of the insurance contract is the determination of the characteristics of the stochastic process representing the market value of the reference fund, which in turns determines the annual return $I_{t}$, that is the "index" underlying the policy ${ }^{20}$.

${ }^{19}$ For a finite difference approach to the valuation of insurance liabilities with ratchet embedded options see Jensen-Jørgensen-Grosen (2001).

${ }^{20}$ As often happens in the investment industry, the insurance company should declare what kind of investment strategy will be followed in the reference fund management. The choice of the asset allocation will have direct effects on the value of the embedded options. 
Since the fund can be composed by bonds and equities, one possibility is to assume:

$$
F_{t}:=\alpha S_{t}+(1-\alpha) W_{t}, \quad 0 \leq \alpha \leq 1,
$$

where $S_{t}$ is a stock index, $W_{t}$ is a bond index and $\alpha$ is here a constant fixed at time zero ${ }^{21}$.

The stock index process is modelled as a geometric Brownian motion, as was implicitly assumed by adopting the BS model for the stock price component of the valuation model. The bond index $W_{t}$ must be chosen as similar as possible to the results of a trading strategy which is considered feasible by the fund manager; hence its characterization requires additional assumptions.

A possible choice is to model $W_{t}$ as the cumulated results of a buy-and-sell strategy, with a fixed trading horizon $\Delta t$, of coupon bonds with a fixed Macaulay duration $D^{\mathrm{MC}} \geq \Delta t$. The valuation procedure is performed by simulating the trading strategy under the CIR model. The results significantly depend on the assumptions on $D^{\mathrm{MC}}$ and $\Delta t$. For a short-term roll-over strategy (e.g. $\Delta t=D^{\mathrm{MC}}=3$ months), $W_{t}$ displays smooth sample paths with high dispersion in the long run. On the other hand, for a buy-and-sell strategy of coupon bonds with mid/long duration ( $\Delta t=3$ months, $D^{\mathrm{MC}}=4,10$ years), the sample paths display greater local volatility, but a reduced long-run dispersion.

Even if this effect could be enhanced by the strong degree of mean reversion displayed by the one-factor CIR model, it should be noted that this behavior is consistent with important empirical findings, as the evidence that short-term rates are more volatile than long-term rates.

An illustration of different investment strategies and of their effect on the cost of the embedded put options can be found in De Felice-Moriconi (2002b, pp. $60-62,64-65)$.

\subsection{Valuation during the life of the policy}

In the NUMAT approach the changes in market conditions have straightforward effects on the valuation during the life of the policy. In order to illustrate these effects we need to better specify our notation and slightly extend some definitions. We shall denote by $t$ the current calendar date and by $T$ the calendar date at which the policy matures; hence if $\tau$ denotes the current age of the policy, the issue date is $t-\tau$ and the term of the policy at issue is $n=T+\tau$. The current readjusted value of the sum insured is:

$$
C_{t}=C_{0} \prod_{k=t-\tau+1}^{t}\left(1+\rho_{k}\right),
$$

where $\rho_{k}$ is the readjustment rate in year $k$ defined by (3). Since $C_{t}$ is known at time $t$, we define the stochastic component of the readjustment factor as:

$\overline{{ }^{21} \text { Maintaining }} \alpha$ constant is known as a "constant mix" investment strategy (Perold-Sharpe, 1988). 


$$
\Phi_{t, T}:=\prod_{k=t+1}^{T}\left(1+\rho_{k}\right)
$$

The corresponding valuation factor at time $t$ will be given by:

$$
u(t, T):=V\left(t ; \Phi_{t, T}\right)=\mathbf{E}_{t}^{Q}\left[e^{-\int_{t}^{T} r_{s} d s} \Phi_{t, T}\right]
$$

which extends the definition (18). The definition of the stochastic reserve $V_{t}$ and of the VBIF $E_{t}$ at time $t$ is straightforward ${ }^{22}$.

In order to illustrate the financial market effects we simulated the valuation during the life of a policy over an historical period starting from the beginning of the 1990's. An efficient secondary market for Italian government bonds (the MTS market) was opened only on Jan 02, 1990; thus any valuation performed at preceding dates would pose serious problems for the model calibration given the low quality of the available data.

In our simulation we considered a single premium pure endowment contract written on Dec 29, 1989 on a life aged $x=40$ years, with initial sum insured $C_{0}=100$ and term $n=20$ years (hence $T=$ Dec 29, 2009). We chose a technical interest rate $i=3 \%$ and a participation coefficient $\beta=80 \%$. The equity component of the reference fund was $\alpha=10 \%$ and the volatility was $\sigma=20 \%$. The investment strategy of the bond component of the reference fund was specified as a buy-and-sell strategy of coupon bonds with Macaulay duration $D^{\mathrm{MC}}=4$ years with trading period $\Delta t=1$ month .

We performed the valuation at the issue date and on each year-end until Dec 31, 2003, calibrating the risk-neutral parameters of the CIR model on the current market data ${ }^{23}$. The first order survival probabilities ${ }_{T-t} p_{x+\tau}^{\prime}$ were computed using the SIM81 mortality tables at all the valuation dates $t^{24}$ and, under assumption (21), they are equal to the $D$ probabilities ${ }_{T-t} p_{x+\tau}$.

The results of the valuations are reported in table 1 . For each valuation date $t$, it is reported the technical reserve:

$$
R_{t}:=C_{t}(1+i)^{-(T-t)}{ }_{T-t} p_{x+\tau},
$$

the stochastic reserve:

$$
V_{t}:=C_{t} u(t, T)_{T-t} p_{x+\tau},
$$

\footnotetext{
${ }^{22}$ A notation more in line with the actuarial tradition would probably be given by defining $u^{(\tau)}(t, T)$ as the valuation factor at date $\tau$ of a policy with term $T$ years (at issue) and current age $t$. For the policies considered here the valuation factor is independent of the age of the policy, hence our definitions allow to use a simplified notation. More importantly, we prefer to denote the calendar time by $t$ in order to stress the central role of the current date in any marked based valuation; the resulting definitions are more consistent with the interpretation of the policy as a financial product.

${ }^{23}$ For the valuation at the issue date we effectively used market data as of Jan 9, 1990, since prices quoted in the first week of MTS's operation were probably not fully reliable.

${ }^{24}$ SIM81 are mortality tables for the general male population, published by ISTAT (Istituto Italiano di Statistica) in 1986.
} 
TABLE 1

VALUATION DURING THE LIFE OF THE POLICY. PUT DECOMPOSITION

\begin{tabular}{lcccccccc}
\hline \hline val. date & $T-t$ & $R_{t}$ & $V_{t}$ & $E_{t}$ & $E_{t} \%$ & $B_{t}$ & $O_{t}^{P}$ & $O_{t}^{P_{\%}}$ \\
\hline Dec 29, 1989 & 20 & 47.88 & 28.54 & 19.34 & 40.40 & 28.39 & 0.15 & 0.51 \\
Dec 31, 1990 & 19 & 50.13 & 30.89 & 19.25 & 38.40 & 30.76 & 0.13 & 0.42 \\
Dec 31, 1991 & 18 & 52.40 & 35.35 & 17.05 & 32.53 & 35.19 & 0.16 & 0.45 \\
Dec 31, 1992 & 17 & 54.70 & 37.25 & 17.45 & 31.91 & 37.14 & 0.10 & 0.28 \\
Dec 31, 1993 & 16 & 57.04 & 45.40 & 11.65 & 20.41 & 43.89 & 1.51 & 3.31 \\
Dec 30, 1994 & 15 & 59.41 & 43.33 & 16.08 & 27.07 & 42.77 & 0.56 & 1.29 \\
Dec 29, 1995 & 14 & 61.81 & 47.57 & 14.24 & 23.04 & 47.12 & 0.45 & 0.95 \\
Dec 31, 1996 & 13 & 64.25 & 55.88 & 8.36 & 13.02 & 53.21 & 2.67 & 4.78 \\
Dec 31, 1997 & 12 & 66.71 & 64.06 & 2.65 & 3.98 & 58.57 & 5.49 & 8.57 \\
Dec 30, 1998 & 11 & 69.21 & 73.13 & -3.92 & -5.66 & 63.26 & 9.87 & 13.50 \\
Dec 30, 1999 & 10 & 71.75 & 69.42 & 2.34 & 3.25 & 64.38 & 5.04 & 7.26 \\
Dec 29, 2000 & 9 & 74.33 & 71.34 & 2.99 & 4.02 & 67.72 & 3.62 & 5.08 \\
Dec 28, 2001 & 8 & 76.96 & 74.15 & 2.81 & 3.65 & 71.05 & 3.11 & 4.19 \\
Dec 31, 2002 & 7 & 79.64 & 79.87 & -0.22 & -0.28 & 75.08 & 4.79 & 5.99 \\
Dec 31, 2003 & 6 & 82.38 & 83.00 & -0.62 & -0.76 & 78.61 & 4.39 & 5.29 \\
\hline \hline
\end{tabular}

TABLE 2

VALUATION DURing THE LIFE OF THE POLICY. CALl DECOMPOSITION

\begin{tabular}{lcccccc}
\hline \hline val. date & $T-t$ & $R_{t}$ & $V_{t}$ & $G_{t}$ & $O_{t}^{C}$ & $O_{t}^{C_{\%}}$ \\
\hline Dec 29, 1989 & 20 & 47.88 & 28.54 & 4.85 & 23.69 & 83.02 \\
Dec 31, 1990 & 19 & 50.13 & 30.89 & 5.99 & 24.90 & 80.62 \\
Dec 31, 1991 & 18 & 52.40 & 35.35 & 10.25 & 25.10 & 71.01 \\
Dec 31, 1992 & 17 & 54.70 & 37.25 & 10.93 & 26.32 & 70.66 \\
Dec 31, 1993 & 16 & 57.04 & 45.40 & 22.62 & 22.78 & 50.18 \\
Dec 30, 1994 & 15 & 59.41 & 43.33 & 15.39 & 27.94 & 64.48 \\
Dec 29, 1995 & 14 & 61.81 & 47.57 & 21.52 & 26.05 & 54.76 \\
Dec 31, 1996 & 13 & 64.25 & 55.88 & 34.82 & 21.07 & 37.70 \\
Dec 31, 1997 & 12 & 66.71 & 64.06 & 47.79 & 16.27 & 25.40 \\
Dec 30, 1998 & 11 & 69.21 & 73.13 & 59.23 & 13.90 & 19.01 \\
Dec 30, 1999 & 10 & 71.75 & 69.42 & 54.56 & 14.86 & 21.41 \\
Dec 29, 2000 & 9 & 74.33 & 71.34 & 59.76 & 11.58 & 16.23 \\
Dec 28, 2001 & 8 & 76.96 & 74.15 & 65.04 & 9.11 & 12.29 \\
Dec 31, 2002 & 7 & 79.64 & 79.87 & 73.94 & 5.93 & 7.42 \\
Dec 31, 2003 & 6 & 82.38 & 83.00 & 78.00 & 5.00 & 6.02 \\
\hline \hline
\end{tabular}


and the corresponding VBIF $E_{t}:=R_{t}-V_{t}$. Since both $R_{t}$ and $V_{t}$ are computed using the SIM81 mortality tables, $E_{t}$ does not include mortality gain and must be thus considered here as the value of only the investment gains. The put decomposition is also illustrated by reporting the base value $B_{t}$ defined by (26) and the value of the embedded put $O_{t}^{P}:=V_{t}-B_{t} . E_{t} \%$ is the VBIF as a percentage of the technical reserve $R_{t} ; O_{t}^{P} \%$ is the put value in percent of the stochastic reserve $V_{t}$. In principle, the scale of $R_{t}$ and $V_{t}$ should be readjusted in each year by the current value of the insured capital $C_{t}$. Since we are mainly interested in the difference between $R_{t}$ and $V_{t}$, we maintained the initial scale of $C_{0}$, thus avoiding arbitrary and irrelevant assumptions on the $C_{t}$ sample path.

Table 2 illustrates the call decomposition. The value of the minimum guaranteed terminal benefit:

$$
G_{t}:=C_{t} v(t, T)_{T} p_{x}
$$

defined in section 3.3 is reported, together with the call price $O_{t}^{C}:=V_{t}-G_{t}$, both in absolute value and in percent of the stochastic reserve.

The results are also reported in figure 1 which illustrates the evolution of $R_{t}$ (solid line), $V_{t}$ (the solid line with bullets), $B_{t}$ (dashed line with circles) and $G_{t}$ (dashed line with stars).

The time evolution of the stochastic reserve is strongly dependent on the historical trend of the bond market. The yield curve was very high in the early 1990's but during the years of the convergence to Euro the Italian bond market experienced a continuous decrease of interest rates. Accordingly, the policy was very profitable at the issuance, when the (financial) embedded value was $40.4 \%$ of the initial reserve and the cost of minimum guarantee was negligeable, the embedded put being far out-of-the-money. Due to the fall in market rates, the put value raised at the $13.5 \%$ of the stochastic reserve at the end of 1998 , causing a negative value of the $\mathrm{VBIF}^{25}$. The time evolution of the call price is in some sense opposite to that of the put price. The call option embedded in the policy was deep in-the-money in the early 1990's and became nearat-the-money in more recent years, as indicated by the $G_{t}$ path moving closer to the $B_{t}$ path. One can observe that a model unable to capture the value of the embedded options would have produced a value of $V_{t}$ close to the base value, so no deficit situation would have been emerged in this example. It is also worthwhile to observe that the variability of the stochastic reserve $V_{t}$ is largely due to the variability of the option components.

\subsection{Issuing policies under different market conditions}

The effects of the current financial market conditions on the policy valuation can be also illustrated by considering the issuance of the same policies at different

${ }^{25}$ In many cases the value of the reference fund $F_{t}$ is defined by accounting rules allowing assets classified as held-to-maturity to be valued by an amortization rule which produces "off-market" prices. This can allow the fund manager to perform some kind of intertemporal smoothing of the returns $I_{t}$ and to spread an annual deficit over more than one year. 


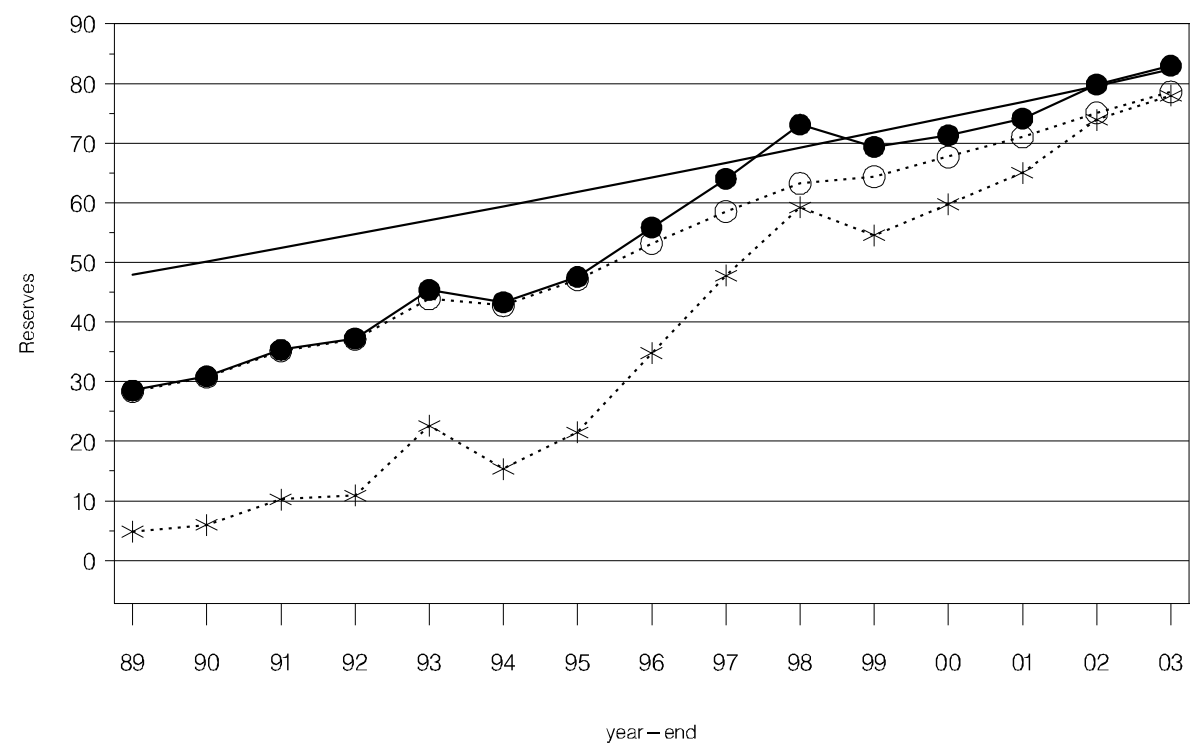

FigurE 1. Time evolution of reserves and of their components.

calendar dates. Let us consider single premium pure endowment contracts with term $n$ years, technical interest rate $i=3 \%$ and participation coefficient $\beta=80 \%$. We assume again an equity component of the reference fund $\alpha=10 \%$ with a volatility $\sigma=20 \%$ and a buy-and-sell strategy for the bond component with $D^{\mathrm{MC}}=4$ years and trading period $\Delta t=1$ month. We assume to have issued these policies for term $n=1,2, \ldots, 20$ years, at different historical dates $t$. For simplicity, we consider only the financial component of the policies, assuming ${ }_{n} p_{x}=1$ for all $t$.

For each valuation date $t$ we calibrated the CIR model on the current market data and then computed the valuation factors:

$$
u(t, t+n):=V\left(t ; \Phi_{t, t+n}\right)=\mathbf{E}_{t}^{Q}\left[e^{-\int_{t}^{t+r_{r} d s}} \Phi_{t, t+n}\right], \quad n=1,2, \ldots, 20 .
$$

For illustration purposes, we also computed the "term structure" of the valuation rates, defined by:

$$
j(t, t+n):=\left[\frac{1}{u(t, t+n)}\right]^{\frac{1}{n}}-1, \quad n=1,2, \ldots, 20 .
$$

This valuation term structure can be compared with the term structure of the interest rates currently prevailing on the market, which is given by:

$$
i(t, t+n):=\left[\frac{1}{v(t, t+n)}\right]^{\frac{1}{n}}-1, \quad n=1,2, \ldots, 20 .
$$


where, as usual, $v(t, T)$ is the market price at the date $t$ of a unit ZCB maturing at time $T$.

We performed the valuation for three different dates:

- $t=\mathrm{Jan}$ 09, 1990, a week after the MTS market was opened;

- $t=$ Dec 30,1998, when the convergence process to Euro was completed;

- $t=\operatorname{Dec} 31,2003$.

In table 3 are plotted, for each of the three issue dates $t$, the term structure of the valuation rates $j(t, t+n)$ with solid line and the term structure of market interest rates $i(t, t+n)$ with dashed line. The flat curve corresponding to the technical rate $i=3 \%$ is also reported with dotted line.

The results can be commented by similar arguments as in the previous example. At the beginning of 1990 (first figure) the market yield curve was at a very high level, ranging from $14 \%$ for $n=1$ to $15.5 \%$ for $n=20$ years; as compared with these market returns, the 3\% technical rate was decidedly low, implying an high level of the investment gains and a low cost of the embedded put options. These effects are summarized by the difference between the $j$ curve and the $3 \%$ flat line; this gap is the counterpart, in the interest rate language, of the VBIF, given by $E_{t}=R_{t}-V_{t}$ : the higher the difference $j(t, t+n)-i$ for a currently issued policy with term $n$, the higher the corresponding $E_{t}$.

In the years 1995-98 the decrease of interest rates was quite dramatic. As illustrated in the second figure of table 3, at the end of 1998 the market rates were ranging from $3 \%$ for $n=1$ to $5.4 \%$ for $n=20$ years. Issuing the policies in this market environment would imply a negative value of $E_{t}$, since the embedded put options would be near at-the-money and the valuation rates would result to be lower than the $3 \%$ level for all the maturities.

The market yield curve was not very different at the end of 2003 (see the third figure). However the situation for the policies as issued at this date would be slightly better; this is essentially due to a volatility effect, since the volatility curve estimated on the market resulted to be lower than the corresponding curve estimated at the end of 1998.

\subsection{Portfolio valuations}

When a real portfolio of outstanding policies has to be analyzed, the situation is obviously much more complex than in the simple examples just considered. An important portion of the policies in force is usually given by participating contracts with constant annual premiums. In these cases the readjustment rule (2) for the benefits must be only partially applied since only the excess return on the investment of the saving premium can be credited to the policyholder. Therefore, the valuation factors at time $t$ will be functions $u^{(x, \tau)}(t, T)$ also depending on the age $x$ of the life insured and on the current age $\tau$ of the policy. Moreover, in many policies benefits payable in case of death are different from benefits payable if the insured is alive; this requires the computation of separated streams of valuation factors. 
issue date: Jan 09, 1990

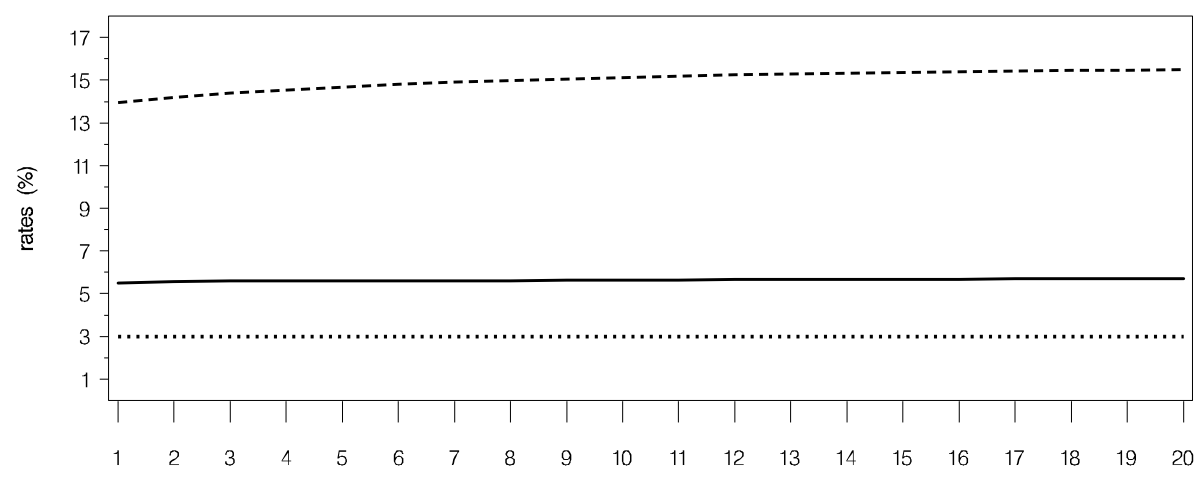

issue date: Dec 30, 1998

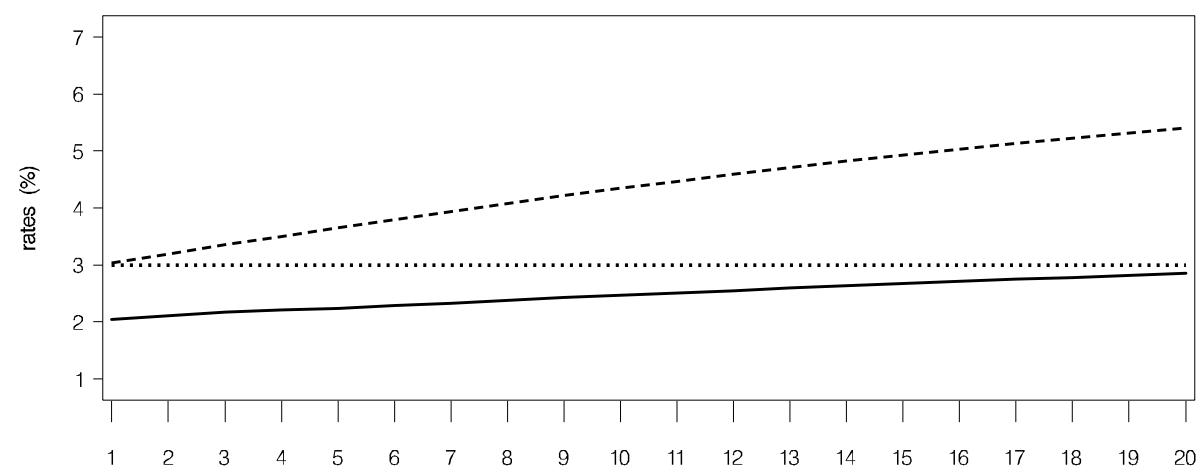

issue date: Dec 31, 2003

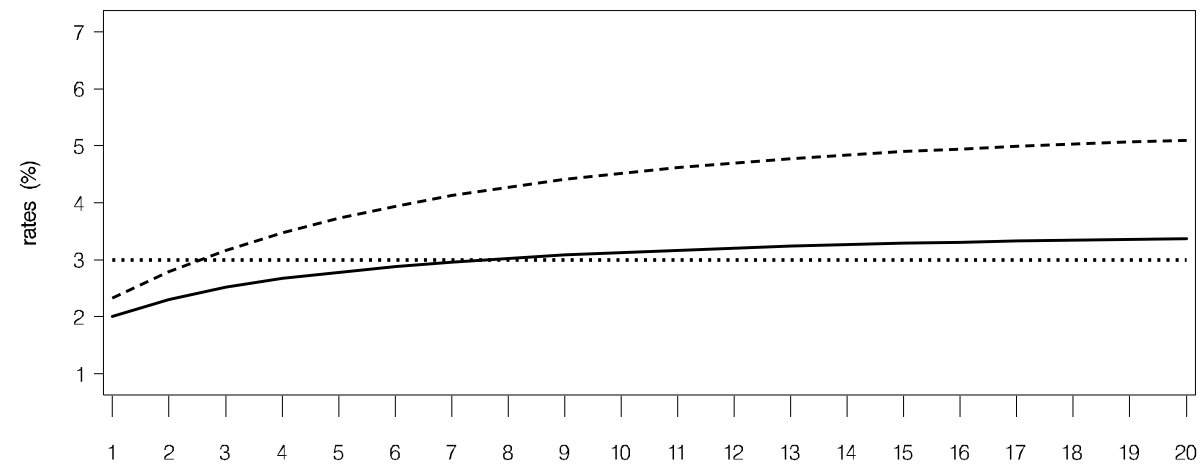

TABLE 3. Term structures of valuation rates at different issue dates. 
However, like for the simple pure endowment policy we can pursue the same approach for any policy of life assurance. It is sufficient to decompose the policy into single premium components with benefits (for survival and/or death and/or other causes) at single time points. For these components we have the liabilities:

$$
Y_{T}:=C_{T} \rrbracket_{\mathcal{G}(x, T)},
$$

where $C_{T}$ is the sum to be eventually paid at time $T$ and $\mathcal{G}$ denotes the exit due to survival, death or other cause. Under the assumption of complete market, the stochastic payoff $C_{T}$ can be replicated by a trading strategy with market price at time $t$ equal to $C_{t} u^{(x, \tau)}(t, T)$, where $C_{t}$ is the current value of the benefit. The same approach must be applied to a premium to be paid at time $T$; of course for constant, i.e. non readjusted, premiums the appropriate valuation factor will be given simply by $v(t, T)$.

In our framework, under the assumption of independence between financial and technical uncertainty, the fair value at time $t$ of $Y_{T}$ is given by:

$$
V_{t}:=V\left(t ; Y_{T}\right)=C_{t} u^{(x, \tau)}(t, T) \mathbf{E}_{t}^{D}\left[\mathbb{G}_{\mathcal{G}(x, T)}\right],
$$

where the $D$ measure discussed in section 2.4 should reflect any technical risk loading. This expression generalizes (19). Summing over all components of all policies in force in the outstanding portfolio we obtain the total net fair value $V_{t}^{\text {Tot }}$ of benefits and premiums, that is the stochastic reserve at time $t$. Extending the definitions in section (3.1), the total VBIF at time $t$ is given by $E_{t}^{T o t}:=$ $R_{t}^{\text {Tot }}-V_{t}^{T o t}$, where $R_{t}^{\text {Tot }}$ is the technical reserve of the portfolio.

In practical applications we usually avoid to perform the valuation using a representative portfolio composed by a reduced number of "model points", since this can produce a number of approximations not easily controllable. Thus the number of valuation factors to be calculated can be very high. Since the Monte Carlo computation of the valuation factors is time consuming, it is required that the calculation procedures are properly optimized.

As concerning portfolios of unit-linked policies, the market based valuation is straightforward. Some computational problems can arise in the valuation of the embedded options for policies with maturity guarantees. Many recently issued index-linked policies are backed by a specified security, typically a "structured bond". Usually these securities have a very complex payoff, possibly containing exotic options; moreover it can happen that their price is quoted on a non efficient market. Thus an appropriate pricing model is needed in order to control possible deviations of the quoted price from the fair value of this product.

\section{Managing the VALUATION VARIABILITY}

The high time variability is obviously a natural characteristic of any market based valuation. This is of course a consequence of a more detailed and upto-date information and is not a problem per se, but rather an advantage of the mark-to-market approach. 
When purely financial products are concerned (as well as many types of index-linked life insurance policies), the timeliness in pricing is crucial, since, given the high liquidity of the markets, any mispricing can expose the issuer to riskless arbitrage; moreover, the issued contract must be immediately hedged at the current market conditions. For traditional life insurance products this problems seems to be not so relevant and a continuous stream of market informations can be considered excessive, or useless, for premium calculation.

Also accounting procedures do not benefit from strongly time-dependent valuation methods; in this kind of applications an important issue is the comparability of the valuation results between different firms. The problem of an increase in volatility of earnings in the accounting reports has widely been recognized, also in the perspective of the new International Accounting Standards (IAS) (see e.g. Morgan Stanley, 2002); these difficulties could be probably overcome by defining some standardized rules for providing an intertemporal smoothing of the operating results.

However, having a better information cannot be a problem; the real issue is how to organize and how to correctly use this information. As pointed out by Bühlmann (2003a), this is particularly true for some applications, as the embedded value measurement. In this case a more detailed analysis of the results given by the market based valuations can provide a better understanding of the value creation mechanisms.

As an example, let us slightly extend our notation of stochastic reserve, by defining:

$$
V_{t}^{(s)}:=V^{(s)}\left(t ; Y_{T}\right), \quad t, s \leq T
$$

as the value of $Y_{T}$ at time $t$ computed under the market conditions prevailing at time $s$; that is $V_{t}^{(s)}$ is the value of the policy with remaining term $T$ - $t$ derived using the parameter vector $\hat{\mathbf{p}}$ calibrated on the market data at time $s$. Of course $V_{t}^{(t)}=V_{t}$. Referring to a time interval $[t, t+\Delta t]$, one can consider the decomposition:

$$
\Delta V_{t}:=V_{t+\Delta t}-V_{t}=\Delta V_{t}^{A}+\Delta V_{t}^{M}, \quad 0 \leq t, t+\Delta t \leq T
$$

where:

- $\Delta V_{t}^{A}:=V_{t+\Delta t}^{(t)}-V_{t}$ is the change in value due to the aging of the policy, independently of changes in market conditions,

and:

- $\Delta V_{t}^{M}:=V_{t+\Delta t}-V_{t+\Delta t}^{(t)}$ is the change in value caused only by the market movements.

This expression suggests an analogous decomposition for the "embedded value earnings":

$$
\Delta E_{t}:=E_{t+\Delta t}-E_{t}=\left(R_{t+\Delta t}-V_{t+\Delta t}\right)-\left(R_{t}-V_{t}\right)
$$

since the change $R_{t+\Delta t}-R_{t}$ is unaffected by market movements. For more details see De Felice-Moriconi (2002a). 


\section{ACKNOWLEDGMENTS}

The authors are greatly indebted to Hans Bühlmann for many helpful and stimulating discussions on the topics of the NUMAT approach. They also wish to express their gratitude to Gilberto Castellani for his invaluable comments and suggestions. Constructive suggestions and helpful comments by two anonymous referees are also gratefully aknowledged.

\section{REFERENCES}

Aase, K.K. and Persson, S.-A. (1994) Pricing of Unit-linked Life Insurance Policies. Scandinavian Actuarial Journal, 1, 26-52.

Aase, K.K. and Persson, S.-A. (2003) New Econ for Life Actuaries. ASTIN Bulletin, 33(2), 117-122.

Bacinello, A.R. (2001) Fair Pricing of Life Insurance Participating Policies with a Minimum Interest Rate Guaranteed. ASTIN Bulletin, 31(2), 275-297.

BACINELlo, A.R. and ORTu, F. (1993) Pricing equity-linked life insurance with endogenous minimum guarantees. Insurance: Mathematics and Economics, 12, 245-257.

Baumgartner, G., Bühlmann, H. and Koller, M. (2004) Multidimensional valuation of life insurance policies and fair value. Mitteilungen der Vereinigung Schweizerischer Versicherungsmathematiker, to appear.

BoyLE, P.P. (1978) Imunization under Stochastic Models of the Term Structure. Journal of the Institute of Actuaries, 105.

BOYLE, P.P. and HARDY, M.R. (1997) Reserving for maturity guarantees: Two approaches. Insurance: Mathematics and Economics, 12, 245-257.

Boyle, P.P. and SChWARTZ, E.S. (1977) Equilibrium prices of guarantees under equity-linked contracts. Journal of Risk and Insurance, 44, 639-660.

Brennan, M.J. and Schwartz, E.S. (1976) The pricing of equity-linked life insurance policies with an asset value guarantee. Journal of Financial Economics, 3, 195-213.

BühlmanN, H. (1995) Life Insurance with Stochastic Interest Rates. In Financial Risk in Insurance (ed. G. Ottaviani), 1-24, Springer, Berlin.

BühlmanN, H. (2002) New Math for Life Actuaries. ASTIN Bulletin, 32(2), 209-211.

Bühlmann, H. (2003a), Comment on the Discussion Article by Aase and Persson. ASTIN Bulletin, 33(2), 123-124.

Bühlmann, H. (2003b) On Teaching Actuarial Science British Actuarial Journal, 9, III.

CAS TASK FORCE (2000) White paper on fair valuing property/casualty insurance liabilities, Casualty Actuarial Society.

Castellani, G., De Felice, M. and Moriconi, F. (1992) Asset-Liability Management. Semideterministic and Stochastic Approach. Transactions of the 24rd International Congress of Actuaries, Montreal, Canada.

Cox, J.C., Ingersoll, J.E. and Ross, S.A. (1985) A Theory of the Term Structure of Interest Rates. Econometrica, 53, 385-406.

De Felice, M. and Moriconi, F. (2002a) Finanza dell'assicurazione sulla vita. Principî per l'asset-liability management e per la misurazione dell'embedded value. Giornale dell'Istituto Italiano degli Attuari, LXV, 13-89.

De Felice, M. and Moriconi, F. (2002b) A Course on Finance of insurance. Groupe Consultatif Actuariel Europeen - Summer School 2002, Milano; reprinted on the websites www. GCActuaries. org/events.html, www.italian-actuaries.org/gruppoconsultivo.htm.

De Felice, M. and Moriconi, F. (2003), Risk Based Capital in P\&C Loss Reserving or Stressing the Triangle. Working Paper n. 1, Research Group on "Insurance Companies and Pension Funds. Valuation Models and Asset-Liability Management", March.

De Felice, M., Moriconi, F. and SAlvemini, M.T. (1993), Italian treasury credit certificates (CCTs): theory, practice, and quirks. BNL Quarterly Review, 185, 127-168.

Delbaen, F. (1990) Equity-linked policies. Bulletin Association Royal Actuaries Belges, 33-52.

Duffie, D. (1992) Dynamic Asset Pricing Theory. Princeton University Press, Princeton, NJ, USA. 
Dybvig, P.H. and Ross, S.A. (1987) Arbitrage. In The New Palgrave. A Dictionary of Economics (eds. J. Eatwell, M. Milgate and P. Newman), Macmillan, London.

Feller, W. (1951) Two Singular Diffusion Problems. Annals of Mathematics, 54, 173-182.

Grosen, A. and Jørgensen, P.L. (1997) Valuation of Early Exercisable Interest Rate Guarantees. The Journal of Risk and Insurance, 64(3), 481-503.

Grosen, A. and Jørgensen, P.L. (2000) Fair valuation of life insurance liabilities: the impact of interest rate guarantees, surrender options and bonus policies. Insurance: Mathematics and Economics, 26, 37-57.

Jensen, B., Jørgensen, P.L. and Grosen, A. (2001) A Finite Difference Approach to the Valuation of Path Dependent Life Insurance Liabilities. Geneva Papers on Risk and Insurance Theory, 26, 57-84.

Karlin, S. and Taylor, H.M. (1981) A Second Course in Stochastic Processes. Academic Press, New York.

Le Roy, S.F. (1987) Present value. In The New Palgrave. A Dictionary of Economics (eds. J. Eatwell, M. Milgate and P. Newman), Macmillan, London.

Miltersen, K.R. and Persson, S.-A.(1999) Pricing rate of return guarantees in a Heath-JarrowMorton framework. Insurance: Mathematics and Economics, 25, 307-325.

Miltersen, K.R. and Persson, S.-A. (2003) Guaranteed Investment Contracts: Distributed and Undistributed Excess Return. Scandinavian Actuarial Journal, 4, 257-279.

Morgan STANLey (2002) Revolution. Equity Research, January 31.

MORICONI, F. (1994) Un modello stocastico bivariato per tassi di interesse nominali e reali. Working Paper n. 16, Research Group on "Modelli per la finanza matematica".

Moriconi, F. (1995) Analyzing Default-Free Bond Markets by Diffusion Models. In Financial Risk in Insurance (ed. G. Ottaviani), 23-46, Springer, Berlin.

Norberg, R. (1999) A theory of bonus in life insurance. Finance and Stochastics, 3(4), 373-390.

PACATI, C. (2003), Financial Valuation of a New Generation Participating Life-Insurance Contract. Proceedings of the VI Spanish-Italian Meeting on Financial Mathematics, Trieste, July 3-5.

Perold, A.F. and Sharpe, W.F. (1988) Dynamic Strategies for Asset Allocation. Financial Analysts Journal, 44, 16-27.

Persson, S.-A. and Aase, K.K.(1997) Valuation of Minimum Guaranteed Returns Embedded in Life Insurance Products. The Journal of Risk and Insurance, 64(4), 599-617.

VENTER, G.G. (1991) Premium calculation implications of reinsurance without arbitrage. ASTIN Bulletin, 21, 223-230.

WANG, S.S. (1996) Premium calculation by transforming the layer premium density. ASTIN Bulletin, 26, 213-234.

WANG, S.S. (2000) A class of distortion operators for pricing financial and insurance risks. Journal of Risk and Insurance, 67, 15-36.

WANG, S.S. (2002) A universal framework for pricing financial and insurance risks, ASTIN Bulletin, 32, 71-92.

Massimo De Felice

Dipartimento di Scienze Attuariali e Finanziarie

Università di Roma "La Sapienza"

via Nomentana, 41

00161 Roma, Italy

E-mail:massimo.defelice@uniromal.it

FRANCO MORICONI

Dipartimento di Economia - Facoltà di Economia

Università di Perugia

via A. Pascoli, 1

06100 Perugia, Italy

E-mail:moriconi@unipg.it

fax: +39 (0)755855221 